\title{
Bundles of acceleration on Banach manifolds
}

Dodson, CTJ and Galanis, GN 2005

Manchester Institute for Mathematical Sciences

School of Mathematics

The University of Manchester

\footnotetext{
Reports available from: http://eprints.maths.manchester.ac.uk/

And by contacting: The MIMS Secretary

School of Mathematics

The University of Manchester

Manchester, M13 9PL, UK
} 


\title{
Bundles of acceleration on Banach manifolds
}

\author{
C.T.J. Dodson ${ }^{a}$, G.N. Galanis ${ }^{b}$ \\ aDepartment of Mathematics, \\ UMIST, \\ Manchester, M60 1QD, United Kingdom \\ E-mail: dodson@umist.ac.uk \\ ${ }^{\mathrm{b}}$ Section of Mathematics, \\ Naval Academy of Greece, \\ Xatzikyriakion, Piraeus 185 39, Greece \\ E-mail: ggalanis@snd.edu.gr
}

We consider an infinite dimensional manifold $M$ modelled on a Banach space $\mathbb{E}$ and we construct smooth fiber bundle structures on the tangent bundle of order two $T^{2} M$, which consists of all smooth curves of $M$ that agree up to their acceleration, as well as on the corresponding second order frame bundle $L^{2} M$. These bundles prove to be associated with respect to the identity representation of the general linear group $G L(\mathbb{E})$ that serves as the structure group of both of them. Moreover, a bijective correspondence between linear connections on $T^{2} M$ and connection forms of $L^{2} M$ is revealed.

\section{Introduction}

In classical differential geometry the fundamental achievement was the encapsulation of curvature and curvature-related linear operators in invariant form. In classical analytic dynamics the corresponding entity is acceleration and its associated linear differential operators, through which trajectories are classified. Whereas the tangent vectors of curves and velocities of particles naturally form vector bundles, their derivatives, which yield curvatures and accelerations, do not.

On the other hand, in the study of fiber (vector or principal) bundles one of the most convenient ways to handle the relevant geometric entities is the use of the notion of associated bundles. To be more precise, consider $P$ a principal bundle with structure group $G$ over the smooth manifold $B$. Given a representation $\rho: G \rightarrow G L(\mathbb{E})$ of $G$ to the general linear group of a space $\mathbb{E}$, we can construct the smooth vector bundle $E:=P \times_{\rho} \mathbb{E}$, as the quotient space of the action $(p, u) \cdot g=\left(p \cdot g, \rho\left(g^{-1}\right)(u)\right)$ of $G$ on (the right) of $P \times \mathbb{E}$. Then, a bijective correspondence can be established between the linear connections of $E$ and the connection forms of $P$ giving, thereby, the opportunity to transfer the study of several geometric entities of the vector bundle $E$ to $P$ and vice versa (for a detailed presentation we refer to [3], [7]).

The most common example of the previous methodology is the bundle of linear frames 
$L(M)=\cup_{x \in M} \mathcal{L} i s\left(\mathbb{E}, T_{x} M\right)$ of a smooth manifold $M$ modelled on $\mathbb{E}$ (where $T_{x} M$ stands for the tangent space of $M$ over $x$ ). In this case the tangent bundle $T M$ of $M$ is associated with $L(M)$ by the trivial representation of $G L(\mathbb{E})$ and every connection of $M$ obtains a counterpart in the, richer, environment of a principal bundle.

In the present note we investigate the possibility of an analogous construction for the second order tangent bundle $T^{2} M$ of a smooth manifold $M$ which consists of all smooth curves of the latter that agree up to acceleration. The former obtains the structure of a smooth vector bundle provided that $M$ is endowed with a linear connection (see [4]).

In previous work ([6]) the first author has studied the case of finite dimensional manifolds by proving that a principal bundle associated to $T^{2} M$ can be obtained. In these results, the manifolds $M$ that admit linear connections are precisely the paracompact ones. Manifolds with connections form a full subcategory $\operatorname{Man} \nabla$ of the category Man of smooth manifolds and smooth maps; the constructions in the above theorems ([6]) provide a functor $\operatorname{Man} \nabla \longrightarrow \operatorname{VBun}([5])$. One of the ways to view a linear connection is as a splitting of $T L M$, which then induces splitting in $J^{2} M$ (called a dissection by Ambrose et al. [1]) and we get also a corresponding splitting in $T^{2} L^{2} M$. This work is based, however, essentially on the (finitely many) local coordinates of $M$, a fact that puts in question the extension of the corresponding results within the framework of infinite dimensional manifolds.

Here, motivated by the increasing use of infinite dimensional manifolds in theoretical Physics (see e.g. [2]), we propose a new coordinate-free approach that allows us to define a second order bundle of linear frames for every infinite dimensional manifold $M$ modelled on a Banach space $\mathbb{E}$. This bundle proves to be associated with $T^{2} M$ via the identity representation of the general linear group of $\mathbb{E} \times \mathbb{E}$. Moreover, a one to one correspondence between linear connections on $T^{2} M$ and the connection forms on the new frame bundle is revealed.

\section{Preliminaries}

In order to make this note self-contained, we present in this preliminary section the differential structure of the second order tangent bundle for a smooth infinite dimensional manifold. For the full exposition of these notions we refer the reader to [4].

Let $M$ be a smooth infinite dimensional manifold modelled on a Banach space $\mathbb{E}$ whose local structure is defined by an atlas $\left\{\left(U_{\alpha}, \psi_{\alpha}\right)\right\}_{\alpha \in I}$. With respect to this collection of local charts of $M$ we construct the corresponding atlas $\left\{\left(\pi_{M}^{-1}\left(U_{\alpha}\right), \Psi_{\alpha}\right)\right\}_{\alpha \in I}$ of the tangent bundle $T M$ of $M$ given by

$$
\Psi_{\alpha}: \pi_{M}^{-1}\left(U_{\alpha}\right) \longrightarrow \psi_{\alpha}\left(U_{\alpha}\right) \times \mathbb{E}:[f, x] \longmapsto\left(\psi_{\alpha}(x),\left(\psi_{\alpha} \circ f\right)^{\prime}(0)\right),
$$

with $[f, x]$ the equivalence class of a smooth curve $f$ of $M$ having $f(0)=x$. The consequential trivializing system of $T(T M)$ is denoted by $\left\{\left(\pi_{T M}^{-1}\left(\pi_{M}^{-1}\left(U_{\alpha}\right)\right), \widetilde{\Psi}_{\alpha}\right)\right\}_{\alpha \in I}$.

Next, provide $M$ with a linear connection $D: T(T M) \rightarrow T M$, which, according to the formalism of Vilms $([8])$, is fully determined by its local forms

$$
D_{\alpha}: \psi_{\alpha}\left(U_{\alpha}\right) \times \mathbb{E} \times \mathbb{E} \times \mathbb{E} \rightarrow \psi_{\alpha}\left(U_{\alpha}\right) \times \mathbb{E} \text { with } D_{\alpha}:=\Psi_{\alpha} \circ D \circ\left(\widetilde{\Psi}_{\alpha}\right)^{-1}, \quad \alpha \in I,
$$


satisfying

$$
D_{\alpha}(y, u, v, w)=\left(y, w+\omega_{\alpha}(y, u) \cdot v\right)
$$

for a family of smooth mappings $\omega_{\alpha}: \psi_{\alpha}\left(U_{\alpha}\right) \times \mathbb{E} \rightarrow \mathcal{L}(\mathbb{E}, \mathbb{E})$ linear with respect to their second variable. The corresponding Christoffel symbols $\left\{\Gamma_{\alpha}\right\}_{\alpha \in I}$ are given by

$$
\Gamma_{\alpha}(y)[u]=\omega_{\alpha}(y, u),(y, u) \in \psi_{\alpha}\left(U_{\alpha}\right) \times \mathbb{E},
$$

and they satisfy the following compatibility condition:

(1) $\left\{\begin{array}{c}\Gamma_{\alpha}\left(\sigma_{\alpha \beta}(y)\right)\left(d \sigma_{\alpha \beta}(y)(u)\right)\left[d\left(\sigma_{\alpha \beta}(y)\right)(v)\right]+\left(d^{2} \sigma_{\alpha \beta}(y)(v)\right)(u)= \\ =d \sigma_{\alpha \beta}(y)\left(\left(\Gamma_{\beta}(y)(u)\right)(v)\right),\end{array}\right\}$

for all $(y, u, v) \in \psi_{\alpha}\left(U_{\alpha}\right) \times \mathbb{E} \times \mathbb{E}$ where by $\sigma_{\alpha \beta}$ we denote the diffeomorphisms $\psi_{\alpha} \circ \psi_{\beta}^{-1}$ of $\mathbb{E}$.

It is worth noting here that exactly the adaptation of this point of view of connections allows us to proceed with a coordinate-free study, a fact essential within the framework of infinite dimensional manifolds.

With these notations, we can define a vector bundle structure on the second order tangent bundle

$$
T^{2} M:=\underset{x \in M}{\cup} T_{x}^{2} M
$$

of $M$, where $T_{x}^{2} M$ stands for the tangent space of order two over $x$. More precisely,

$$
T_{x}^{2} M:=C_{x} / \approx_{x}
$$

where $C_{x}$ is the set of all smooth curves of $M$ through $x$ and the equivalence relation $\approx_{x}$ is given by

$$
f \approx_{x} g \Leftrightarrow f^{\prime}(0)=g^{\prime}(0) \text { and } f^{\prime \prime}(0)=g^{\prime \prime}(0)
$$

Then, for all $a \in I$ we set $\Phi_{\alpha}: \pi_{2}^{-1}\left(U_{\alpha}\right) \longrightarrow U_{\alpha} \times \mathbb{E} \times \mathbb{E}$ with

$$
\Phi_{\alpha}\left([f, x]_{2}\right)=\left(x,\left(\psi_{\alpha} \circ f\right)^{\prime}(0),\left(\psi_{\alpha} \circ f\right)^{\prime \prime}(0)+\Gamma_{\alpha}\left(\psi_{\alpha}(x)\right)\left(\left(\psi_{\alpha} \circ f\right)^{\prime}(0)\right)\left[\left(\psi_{\alpha} \circ f\right)^{\prime}(0)\right]\right),
$$

where $\pi_{2}: T^{2} M \rightarrow M:[f, x]_{2} \mapsto x$. These mappings form the necessary trivializing family of $T^{2} M$. The corresponding transition functions

$$
T_{\alpha \beta}: U_{\alpha} \cap U_{\beta} \rightarrow \mathcal{L}(\mathbb{E} \times \mathbb{E}, \mathbb{E} \times \mathbb{E}): x \longmapsto \Phi_{\alpha, x} \circ \Phi_{\beta, x}^{-1},
$$

where $\Phi_{\alpha, x}:=\left.p r_{2} \circ \Phi_{\alpha}\right|_{\pi_{2}^{-1}(x)}$ are the restrictions to the fibers, are proved equal with $\left(d \sigma_{\alpha \beta} \circ \psi_{\beta}\right) \times\left(d \sigma_{\alpha \beta} \circ \psi_{\beta}\right)$ for all $\alpha, \beta \in I$. As a result, $T^{2} M$ turns out to be a vector bundle over $M$ with fibers of type $\mathbb{E} \times \mathbb{E}$ and structure group $G L(\mathbb{E} \times \mathbb{E})$. 


\section{Frame bundles of order two for infinite dimensional manifolds}

Based on the notations of the previous section, we study here the notion of the second order frame bundle of a smooth manifold $M$. Our final goal is to equip it with a principal bundle structure so as to be associated with $T^{2} M$ by means of the identity representation of the general linear group of the fiber type.

Let $\mathbb{E}$ be an arbitrarily chosen Banach space.

Definition 2.1. We call the frame bundle of order two of the $\mathbb{E}$-modelled manifold $M$ the union

$$
L^{2}(M):=\cup_{x \in M} \mathcal{L} i s\left(\mathbb{E} \times \mathbb{E}, T_{x}^{2} M\right) .
$$

The first step is to endow this set with a principal bundle structure:

Proposition 2.2. $L^{2}(M)$ is a smooth principal fiber bundle over $M$ with structure group $G L(\mathbb{E} \times \mathbb{E})$.

Proof. Let $\left\{\left(U_{\alpha}, \psi_{\alpha}\right)\right\}_{\alpha \in I}$ be a smooth atlas of $M$ and $\left\{\left(\pi_{2}^{-1}\left(U_{\alpha}\right), \Phi_{\alpha}\right)\right\}_{\alpha \in I}$ the corresponding trivializing cover of $T^{2} M$ as explicitly presented in Preliminaries. We denote then by $\tau_{\alpha, x}:=\left.p r_{2} \circ \Phi_{\alpha}\right|_{\pi_{2}^{-1}(x)} \in \mathcal{L} i s\left(T_{x}^{2} M, \mathbb{E} \times \mathbb{E}\right)$ the corresponding projections to the fibers.

As projection of $L^{2}(M)$ to its basis we define the map $p: L^{2}(M) \rightarrow M$ with $p(h)=x$, for $h$ is an element of $\mathcal{L} i s\left(\mathbb{E} \times \mathbb{E}, T_{x}^{2} M\right)$. On the other hand, the general linear group $G L(\mathbb{E} \times \mathbb{E})$ acts on (the right of) $L^{2}(M)$ in a natural way $h \cdot g:=h \circ g$.

The local structure of the bundle under construction is determined by the mappings

$$
F_{\alpha}: p^{-1}\left(U_{\alpha}\right) \rightarrow U_{\alpha} \times G L(\mathbb{E} \times \mathbb{E}): h \longmapsto\left(p(h), \tau_{\alpha, p(h)} \circ h\right) .
$$

Each of these $F_{\alpha}$ is a well defined bijection. Indeed, the injectivity is a direct consequence of the fact that the $\tau_{\alpha, x}(\alpha \in I)$ are linear isomorphisms. On the other hand, surjectivity follows from the fact that all elements $(x, g) \in U_{\alpha} \times G L(\mathbb{E} \times \mathbb{E})$ can be written in the form $F_{\alpha}\left(\tau_{\alpha, x}^{-1} \circ g\right)$.

Therefore, each $X_{a}:=p^{-1}\left(U_{\alpha}\right), a \in I$, can be endowed with a smooth manifold structure modelled on the Banach space $\mathbb{E} \times G L(\mathbb{E} \times \mathbb{E})$. Checking that the image of every intersection $F_{\alpha}\left(X_{a} \cap X_{b}\right)=\left(U_{\alpha} \cap U_{b}\right) \times G L(\mathbb{E} \times \mathbb{E})$ is open in $F_{\alpha}\left(X_{a}\right)$, we conclude that $X_{a} \cap X_{b}$ is open in $X_{a}$.

Moreover, the differential structure of $X_{a} \cap X_{b}$ as a submanifold of $X_{a}$ coincides with that obtained via $X_{b}$, since the mapping $F_{b} \circ F_{\alpha}^{-1}$ is a diffeomorphism:

$$
\left(F_{b} \circ F_{\alpha}^{-1}\right)(x, g)=\left(x,\left(\operatorname{comp} \circ\left(T_{b a}, i d_{\mathcal{L}(\mathbb{E})}\right)\right)(x, g)\right) .
$$

Here, comp denotes the composition factor $\mathcal{L}(\mathbb{E}) \times \mathcal{L}(\mathbb{E}) \rightarrow \mathcal{L}(\mathbb{E}), i d_{\mathcal{L}(\mathbb{E})}$ is the identical mapping of $\mathcal{L}(\mathbb{E})$ and $\left(T_{b a}\right)_{a, b \in I}$ the transition functions of the second order tangent bundle of $M$.

Thus, based on the well known gluing Lemma ([3; $\left.\left.\mathrm{N}^{o} 5.2 .4\right]\right)$ we conclude that $L^{2}(M)=$ $\cup_{a \in I} X_{a}$ is a principal Banach bundle, as requested, with transition functions

$$
G_{b a}: U_{\alpha} \cap U_{b} \rightarrow G L(\mathbb{E} \times \mathbb{E}): x \longmapsto\left(F_{b, x} \circ F_{\alpha, x}^{-1}\right)
$$


These are related with the corresponding transition functions of $T^{2} M$ via the basic relation:

$$
G_{b a}(x)(g)=\left(F_{b, x} \circ F_{\alpha, x}^{-1}\right)(g)=T_{b a}(x) \circ g .
$$

Based on the previous result, we are in a position now to prove that the second order frame and tangent bundle are always associated for any smooth Banach-modelled manifold. Namely, if we consider the action

$$
(h,(u, v)) \cdot g=\left((h \circ g), g^{-1}(u, v)\right)
$$

of the group $G L(\mathbb{E} \times \mathbb{E})$ on the right of the product $L^{2}(M) \times(\mathbb{E} \times \mathbb{E})$, then the following main result holds:

Theorem 2.3. The quotient $\left.\left(L^{2}(M) \times \mathbb{E} \times \mathbb{E}\right)\right) / G L(\mathbb{E} \times \mathbb{E})$ coincides, up to isomorphism, with $T^{2} M$.

Proof. A local structure on the quotient under consideration can be defined using the atlas $\left\{\left(U_{\alpha}, \psi_{\alpha}\right)\right\}_{a \in I}$ of $M$ and the corresponding one $\left\{\left(p^{-1}\left(U_{\alpha}\right), F_{\alpha}\right)\right\}_{a \in I}$ of $L^{2}(M)$ as follows. Let

$$
\tilde{\pi}: \tilde{E}:=\left(L^{2}(M) \times \mathbb{E} \times \mathbb{E}\right) / G L(\mathbb{E} \times \mathbb{E}) \rightarrow M:[h,(u, v)] \longmapsto p(h)
$$

be the projection on $M$. We define, for each $a \in I$, the mapping:

$$
\widetilde{\Phi}_{\alpha}: \tilde{\pi}^{-1}\left(U_{\alpha}\right) \rightarrow U_{\alpha} \times \mathbb{E} \times \mathbb{E}:[h,(u, v)] \longmapsto\left(p(h),\left(p r_{2} \circ F_{\alpha}\right)(h)(u, v)\right)
$$

where $p r_{2}$ denotes the projection of $U_{\alpha} \times G L(\mathbb{E} \times \mathbb{E})$ to the second factor.

Each $\widetilde{\Phi}_{\alpha}$ is injective since if the classes $[h,(u, v)],\left[h_{1},\left(u_{1}, v_{1}\right)\right]$ coincide through $\widetilde{\Phi}_{\alpha}$, then $p(h)=p\left(h_{1}\right)=x \in M$ and $\left(\tau_{\alpha, x} \circ h\right)(u, v)=\left(\tau_{\alpha, x} \circ h_{1}\right)\left(u_{1}, v_{1}\right)$. As a result, $h(u, v)=h_{1}\left(u_{1}, v_{1}\right)$ and with respect to the isomorphism $g:=h_{1}^{-1} \circ h$ of $\mathbb{E} \times \mathbb{E}$ the classes $[h,(u, v)]$ and $\left[h_{1},\left(u_{1}, v_{1}\right)\right]$ coincide.

Also, each $\widetilde{\Phi}_{\alpha}$ is surjective. Indeed, considering an element $(x,(u, v))$ of $U_{\alpha} \times \mathbb{E} \times \mathbb{E}$ and the isomorphism $h:=\tau_{\alpha, x}^{-1} \in \mathcal{L} i s\left(\mathbb{E} \times \mathbb{E}, T_{x}^{2} M\right)$, we see that $\widetilde{\Phi}_{\alpha}([h,(u, v)])=\left(x,\left(\tau_{\alpha, x} \circ\right.\right.$ $h)(u, v))=(x,(u, v))$.

Finally, it is a matter of routinely checking the fact that the restrictions onto the fibers $\widetilde{\Phi}_{\alpha, x}:=\left.p r_{2} \circ \widetilde{\Phi}_{\alpha}\right|_{\tilde{\pi}^{-1}(x)}$ satisfy the relations $\widetilde{\Phi}_{b, x} \circ \widetilde{\Phi}_{\alpha, x}^{-1}=\tau_{b, x} \circ \tau_{\alpha, x}^{-1}$, and hence they are linear isomorphisms.

Summarizing, the local charts $\left\{\left(U_{\alpha}, \widetilde{\Phi}_{\alpha}\right)\right\}_{a \in I}$ define on $\tilde{E}$ a vector bundle structure with corresponding transition functions

$$
\widetilde{T}_{b a}(x)=\widetilde{\Phi}_{b, x} \circ \widetilde{\Phi}_{\alpha, x}^{-1}=\tau_{b, x} \circ \tau_{\alpha, x}^{-1} ; x \in U_{\alpha} \cap U_{b} .
$$

Proceeding now to the establishment of an isomorphism between this vector bundle and the second order tangent bundle of $M$, we define

$$
G: \tilde{E} \rightarrow T^{2} M:[h,(u, v)] \longmapsto h(u, v) .
$$


This is a well-defined mapping since, for two equivalence classes $[h,(u, v)],\left[h_{1},\left(u_{1}, v_{1}\right)\right]$, with respect to an element $g$ of $G L(\mathbb{E} \times \mathbb{E})$, we have that $h \circ g=h_{1}, g\left(u_{1}, v_{1}\right)=(u, v)$, thus $h(u, v)=h_{1}\left(u_{1}, v_{1}\right)$.

$G$ is also injective since $G([h,(u, v)])=G\left(\left[h_{1},\left(u_{1}, v_{1}\right)\right]\right)$ leads to $h(u, v)=h_{1}\left(u_{1}, v_{1}\right)$ and, with respect to the isomorphism $g:=h_{1}^{-1} \circ h$, we obtain

$$
\left(h_{1},\left(u_{1}, v_{1}\right)\right) \cdot g=\left(h_{1} \circ g, g^{-1}\left(u_{1}, v_{1}\right)\right)=(h,(u, v)) .
$$

Furthermore, $G$ is surjective. Indeed, consider an element $w$ in $T^{2} M$ and more precisely in the fiber $T_{x}^{2} M$; let $\left(U_{\alpha}, \psi_{\alpha}\right)$ be a chart of $M$ through $x,\left(U_{\alpha}, \Phi_{\alpha}\right)$ its counterpart on $T^{2} M$ (as described in the Preliminary section). Then, $w$ can be obtained as the image of $\left(\left.p r_{2} \circ \Phi_{\alpha}\right|_{\pi_{2}^{-1}(x)}\right)^{-1}(u, v)$ through $G$.

We conclude the proof checking that $G$ is a vector bundle isomorphism since it maps the local trivializations of $\tilde{E}$ to those of $T^{2} M$ :

$$
\left.\left(\Phi_{\alpha} \circ G\right)([h,(u, v)])=\Phi_{\alpha}(h(u, v))=\left(p(h),\left(\tau_{\alpha, p(h)} \circ h\right)(u, v)\right)\right)=\widetilde{\Phi}_{\alpha}([h,(u, v)]), a \in I .
$$

\section{Association of geometric properties}

Based on the results presented so far, we prove that a correspondence between linear connections of the second order tangent bundle and connections on the frame bundle of order two can be established. This enables us to transform the study of several geometric entities of $T^{2} M$, like parallel displacement, curvature forms, etc to the richer framework of the principal bundle $L^{2}(M)$.

Theorem 3.1. Every linear connection $\nabla$ of the second order tangent bundle $T^{2} M$ corresponds bijectively to a connection $\omega$ of $L^{2}(M)$.

Proof. Let $\left\{\left(U_{\alpha}, \psi_{\alpha}\right)\right\}_{a \in I}$ be an atlas of $M$ and $\left(\pi_{2}^{-1}\left(U_{\alpha}\right), \Phi_{\alpha}\right),\left\{\left(p^{-1}\left(U_{\alpha}\right), F_{\alpha}\right)\right\}_{a \in I}$ the associated trivializations of $T^{2} M, L^{2}(M)$ respectively (see Preliminaries and Proposition 2.2). We denote by $\left\{s_{\alpha}: U_{\alpha} \rightarrow p^{-1}\left(U_{\alpha}\right): x \mapsto F_{\alpha}^{-1}\left(x, i d_{\mathbb{E} \times \mathbb{E}}\right)\right\}_{a \in I}$ the corresponding natural local sections of $L^{2} M$, where $i d_{\mathbb{E} \times \mathbb{E}}$ stands for the identity map of $\mathbb{E} \times \mathbb{E}$. Any linear connection $\nabla$ of $T^{2} M$ is fully determined by its family of Christoffel symbols $\left\{\Gamma_{\alpha}: \psi_{\alpha}\left(U_{\alpha}\right) \rightarrow \mathcal{L}(\mathbb{E} \times \mathbb{E}, \mathcal{L}(\mathbb{E} \times \mathbb{E}, \mathbb{E} \times \mathbb{E}))\right\}_{a \in I}$ which are defined via the relations $D_{\alpha}(y, u, v, w)=\left(y, w+\Gamma_{\alpha}(y)(u)[v]\right)$, if $D_{\alpha}$ are the local forms of $D$. Based on this family, we may define, for each $a \in I$, the local forms $\omega_{\alpha} \in \Lambda^{1}\left(U_{\alpha}, \mathcal{L}(\mathbb{E} \times \mathbb{E})\right)$ with:

$$
\omega_{\alpha}(x)\left(T_{\psi_{\alpha}(x)} \psi_{\alpha}^{-1}(v)\right)(u)=\left(\left[\Gamma_{\alpha}\left(\psi_{\alpha}(x)\right)\right](u)\right)(v) .
$$

In view of relation (1), we may prove that the $\omega_{\alpha}$ satisfy the compatibility condition

$$
\omega_{\beta}=A d\left(G_{a \beta}^{-1}\right) \cdot \omega_{\alpha}+G_{a \beta}^{-1} \cdot d G_{a \beta},
$$

for every pair of indices $(\alpha, \beta) \in I^{2}$. Here, $d G_{a \beta}$ denotes the total (logarithmic) differential of the transition functions of $L^{2}(M)$ and $A d$ the adjoint representation of the general linear group $G L(\mathbb{E} \times \mathbb{E})$. As a result, a unique connection $\omega$ on $L^{2}(M)$ can be defined satisfying $s_{\alpha}^{*} \omega=\omega_{\alpha}$, where the star denotes the pullback with respect to the section $s_{\alpha}$. 


\section{Open problems}

The results presented in this note are the outcome of an ongoing research within the framework of infinite dimensional manifolds and fiber bundles. We conclude by mentioning some currently open problems:

- Existence of a canonical form on the frame bundle of order two which extends the classical notion of torsion,

- Existence of a universal connection which may "produce" all connections on $T^{2} M$ and $L^{2}(M)$,

- Development of a cohomological classification of tangent and frame bundles of second order.

\section{REFERENCES}

1. W. Ambrose, R.S. Palais and I.M. Singer. Sprays, Anais da Academia Brasileira de Ciencias 32 (1960) 163-178.

2. M.C. Abbati, A. Mania, On differential structure for projective limits of manifolds, J. Geom. Phys. 29 (1999), no. 1-2, 35-63.

3. N. Bourbaki, Varietés différentielles et analytiques, Fascicule de résultats, $§ 1-7$, Hermann, Paris (1967).

4. C.T.J. Dodson and G.N. Galanis, Second order tangent bundles of infinite dimensional manifolds, to appear in J. Geom. Phys.

5. C.T.J. Dodson and M.S. Radivoiovici, Second order tangent structures. International J. Theor. Phys. 21, 2,(1982), 151-161.

6. C.T.J.Dodson and M.S.Radivoiovici, Tangent and Frame bundles of order two, Analele stiintifice ale Universitatii "Al. I. Cuza", 28 (1982), 63-71.

7. W. Greub, S. Halperin and R. Vanstone, Connections, Curvature and Cohomology, Vol. II, Academic Press, N. York 1973.

8. J. Vilms, Connections on tangent bundles, J. Diff. Geom. 41 (1967), 235-243. 